\title{
A Research on the Innovative Cooperation Model of New Silk Road
}

\section{Economic Belt}

\author{
Fengxiang Jiang ${ }^{1, a}$ \\ ${ }^{1}$ Business School, Xi'an Peihua University, Xi'an City, Shaanxi Province, 710125, China
}

\begin{abstract}
Keywords: New Silk Road; Economic Belt; Innovation; Cross-regional Cooperation Model
\end{abstract}
\begin{abstract}
Silk Road Economic Belt is an economic cooperation zone between China and the West Asian countries, which roughly overlaps the ancient Silk Road. It includes five provinces in the northwest, which are Shaxi, Gansu, Qinghai, Ningxia and Xinjiang. In the southwest, it contains four provinces and municipalities, including Chongqing, Sichuan, Yunnan and Guangxi. Based on the author's learning and practical experience, this paper analyzed the innovative cooperation model of New Silk Road Economic Belt, which is the cross-regional cooperation model. Then, the article analyzed the opportunities and challenges in this model. Finally, this paper put forward construction strategies of cross-regional cooperation model of New Silk Road Economic Belt.
\end{abstract}

\section{Introduction}

At present, the recession of the global economy has accelerated the pace of global economic integration and regional economic cooperation to a certain extent. Many countries expect to achieve the objects of economic growth with the cross-regional cooperation. Making a general survey of the world economy today, we find that the Eurasian continent plays a decisive role. New Silk Road Economic Belt proposed by President Xi Jinping attracts the attention of the whole world and gets the positive response from the countries along the economic belt. The construction of New Silk Road Economic Belt is very important to accelerate the cross-regional cooperation in global economy, create a favorable geopolitical and economic environment, find new economic growth point in China, promote the west opening strategy, accelerate the west development and safeguard China's economic growth. Nonetheless, how to build a scientific and rational cross-regional cooperation mechanism and continue to promote the cross-regional cooperation in New Silk Road Economic Belt is an urgent and arduous issue currently, which is just the topic that this paper discussed.

\section{The Current Situation of Cross-regional Cooperation in New Silk Road Economic Belt}

Over the past two decades, the cooperation between China and the governments of Central Asia has become increasingly close and fruitful, which mainly reflects in the following aspects: first, the cooperation and exchanges between political parties have become increasingly frequent, which enhances political mutual trust between China and countries in Central Asian and lays a foundation for the cooperation and exchanges in other fields. Second, the establishment of Shanghai Cooperation Organization (SOE) provides an important platform between China and the Central Asian governments, which plays an very important role in regional trade and investment facilitation, and economic cooperation. China's relevant policies in Central Asia have been developed from nothing and gradually improved. Third, China and Central Asian governments have effective cooperation in anti-drug and security, which curbs terrorist and drug crime.

Cross-regional cooperation between China, Central Asia and Russia has developed for many years under the framework of Shanghai Cooperation Organization and it has achieved some results in government cooperation and business cooperation. However, the existing cooperation level is not rich. There are many government-led cooperation activities, but the civil cooperation is less. In addition, the cooperation mainly is in the energy field, which is not enough in depth and breadth. The intergovernmental cooperation is relatively deep, but the cooperation between enterprises and 
cooperate intermediary organizations is not deep enough. Furthermore, the cooperation form is relatively simple. The main forms are intergovernmental joint meetings and corporate cross-regional investment alliances and there are fewer other ways. On the other hand, this shows there is a huge cooperation potential and space in the future, so the cross-regional cooperation in New Silk Road Economic Belt has many advantages.

\section{Development Opportunities of the Cross-regional Cooperation Model}

At present, China's economic development has provided the best historical opportunity for the construction of New Silk Road Economic Belt. At present, there are huge connective strategic needs in the two major economic circles of the Eurasian continent. China is at the most favorable geographical strategic position and it is one of the most important economic engines in the world. "The world's second largest economy + China model" undoubtedly constitutes a great attraction and centripetal force for Central Asian countries. After the financial crisis, the global economic recovery is still struggling. Countries along the Silk Road Economic Belt and Russia are facing the problem of urgent economic development. Therefore, the common interest demand in the cross-regional cooperation is more important than the differences, so it is the best time to promote the construction of New Silk Road Economic Belt.

At present, Shanghai Cooperation Organization has set up a bank association and it will establish and develop SOE Development Bank and SOE special accounts in the future possibly, which will play a very important role in major cross-regional construction projects financing and high-level financial cooperation, such as monetary policy coordination, cross-regional capital flows and monetary integration. Therefore, the development of Shanghai Cooperation Organization not only provides an important political basis and cooperation experience for the development of New Silk Road Economic Belt, but also lays a good foundation for promoting cross-regional trade and investment, deepening economic and technological cooperation and establishing a free trade zone in the future.

China's domestic economic downward pressure has increased, while the expansion of the west is conducive to increasing the inherent potential and maneuvering space of China's economic development. Affected by global financial crisis and other factors, China's economic growth has slowed down obviously and the downward pressure continues to increase. In this context, to promote the construction of New Silk Road Economic Belt, connect the European economic circle and form another western open economic corridor which is the longest one and has the greatest development potential, will greatly expand the space of China's economic development and increase the inherent potential of China's economic development.

\section{Challenges of the Cross-regional Cooperation Model}

Experience in the construction of cross-regional cooperation mechanisms and policy supply is insufficient. After rapid economic development for more than 30 years, China has gathered a wealth of experience in international trade, but how to build a cross-regional economic cooperation organization and establish a corresponding supporting policy system without political blame from other countries is undoubtedly a huge challenge for China. It is very difficult to do this well and use the appropriate strategic steps to control the area with the most complex and most chaotic religions and culture in the world to promote the construction of cross-regional all-round cooperation mechanism. However, the research and thinking on these problems in current academic circle in China is not mature.

The development of Central Asian countries is affected by many large countries and neighboring countries, and there is some uncertainty about the country in the future. Central Asia is located in the Eurasian connection point and strategic joint, so the geopolitical position is obvious. In particular, the second large railway in Eurasian continent makes Central Asia the important corridor to connect 
traffic between the east and the west. As a result, many large countries or neighboring countries have launched a fierce competition in this region, such as the United States, Russia, Turkey and Iran. They intend to use politic, military, economic assistance, cultural exchanges and diplomacy to exert their influence on these countries, so as to maximize their benefits. However, Central Asia traditionally belongs to Russia's "sphere of influence". Based on global hegemony of the national strategy, the United States takes Central Asia as a vital region for its interest. Turkey's "Turkicism" and Iran's "Islamic fundamentalism" forces have also strengthened their influence on the Central Asian region actively. As a result, the future trend of Central Asia where a variety of forces compete for the strategic point is uncertain, so that the cross-regional cooperation of New Silk Road Economic Belt will face this problem for a long term.

Central Asia is a region where many religions, nationalist ideas and cultures converge and violently collide. There exist many contradictions among Central Asian countries. Their mutual trust is low and they lack mutual benefit and win-win consciousness. In conclusion, this is the region with the most intense collisions in diverse religious ideas and cultures in the world. These complex and varied religious ideologies and cultures and the lower trust between Central Asian countries bring many unstable factors to the cross-regional cooperation of New Silk Road Economic Belt.

\section{Construction Strategies of the Cross-regional Cooperation Model of New Silk Road Economic Belt}

The development stages of the cross-regional cooperation of New Silk Road Economic Belt. In terms of strategic development goals of New Silk Road Economic Belt, it will be an interests community with all-round and cross-regional cooperation in the future, including politics, economy, finance, industry and regional security, which will eventually become the Eurasian destiny community. That means that the cross-regional cooperation framework and its mechanism of New Silk Road Economic Belt will be a complex and giant system engineering. Based on the current development of New Silk Road Economic Belt and the status quo of domestic and international political and economic development, we believe that the construction of cross-regional cooperation mechanism of New Silk Road Economic Belt should adhere to the principle of "the development from near to the distant and from the easy to the difficult". The development stages are successively the initial stage, development stage, expansion stage and formation stage. The goal in the initial stage is to achieve full cross-regional economic cooperation with five Central Asian countries to form the basic model and prototype of cross-regional cooperation of New Silk Road Economic Belt; the object of the development stage is to further improve cross-regional cooperation organization framework and legal agreements of New Silk Road Economic Belt. On the basis of the above goal, China will achieve cooperation with Russia and other neighboring countries; the goal of the expansion stage is to attract neighboring countries along the economic belt to join in and constantly expand cooperation scope and space based on existing cooperation forms and frameworks; the goal of the fourth stage is to basically achieve the cooperation of New Silk Road Economic Belt which crosses the Asia and Europe from the east of Lianyungang to the west of Rotterdam. Finally, the destiny community with more than 30 countries and over 40 million people is basically formed.

The cross-regional cooperation mechanism of New Silk Road Economic Belt. Governments, enterprises and various non-governmental organizations are the most important subjects to promote the cross-regional cooperation of New Silk Road Economic Belt. In order to promote cross-regional cooperation, it is very important to formulate a scientific and reasonable cross-regional cooperation mechanism. Combined with domestic and international experience in cross-regional cooperation and the current development situation and problems of New Silk Road Economic Belt, we believe that the cross-regional cooperation mechanism of New Silk Road Economic Belt should take the relevant governments and enterprises as the main active participants. It is a complete organic system with target mechanism, dynamic mechanism, operating mechanism, innovation mechanism, decision-making mechanism and security mechanism. 
The new mechanism includes two aspects: the innovative mechanism of the regional cooperation framework and the innovative mechanism of the regional cooperation agreements. As an organic system, in order to make cross-regional cooperation adapt to the continuous development and changes of New Silk Road Economic Belt, it is very necessary to enhance innovation researches on cross-regional cooperation framework, system and mechanism with the continuous deepening and expansion of cooperation scope. The innovation of regional cooperation agreements is to study cooperation fields, cooperation agreements and trade rules to propose innovation research reports or suggestions on adjustment and innovation based on the cooperation development, so as to better adapt to or promote the smooth cooperation.

\section{Acknowledgements}

Fund project: Initial results of the project research on the major theoretical and practical issues and important theory and realistic problem in the social sciences of Shaanxi Province, 2016. Belt

Project Name: A Research on the Innovative Cooperation Model of New Silk Road Economic

Project number: $2016 Z 013$

\section{References}

[1] Wang Dan. A Research on the Regional Cooperation Operation Mechanism of New Silk Road Economic Belt [J]. China Journal of Commerce, 2016, (31): 120-122.

[2 Zhang Jianjun, Duan Limin and Zhao Zuoxiang. A Research on the Regional Cooperation Operation Mechanism of New Silk Road Economic Belt [J]. Journal of Xidian University (Social Science Edition), 2016 (04): 87-96.

[3] Wang Yapeng, Gao Yunfei and Gui Helei. A Discussion on the Innovative Regional Cooperation Mechanism of New Silk Road Economic Belt [J]. Brand (on the Second Half of the Month), 2015, (10): 106.

[4] Huai Jianjun, Wang Zhengbing and Zhao Yinke. A Research Review of New Silk Road Economic Belt [J]. Academics in China, 2015, (01): 219-228 +327-328.

[5] Zhang Handong. A Connection with New Silk Road [J]. Journal of Zhejiang , 2014, (12): 30-31.

[6] Shao Yuqun. The Plan Assessment of American Connection with New Silk Road [J]. South Asian Studies, 2014, (02): 58-70 + 158. 